\title{
Existence and Born-Oppenheimer Asymptotics of the Total Scattering Cross-Section in Ion-Atom Collisions
}

\author{
Thierry Jecko \\ Université de Rennes I \\ Département de Mathématiques \\ F-35042 Rennes Cedex, FRANCE \\ e-mail: jecko@maths.univ-rennes1.fr
}

\author{
Markus Klein \\ Universität Potsdam \\ Institut für Mathematik \\ D-14469 Potsdam, GERMANY \\ e-mail: mklein@math.uni-potsdam.de
}

\author{
Xue Ping Wang \\ Université de Nantes \\ Département de Mathématiques \\ F-44072 Nantes Cedex, FRANCE \\ e-mail: Xue-Ping.Wang@math.univ-nantes.fr
}

\begin{abstract}
We prove the finiteness of the total scattering cross-section for ion-atom collisions with an initial channel given by a simple eigenvalue of the internal Hamiltonian describing the neutral cluster, i.e. the atom. Under more restrictive assumptions, we show that some effective interaction in Born-Oppeheimer approximation is precisely of order $\mathcal{O}\left(|x|^{-4}\right)$ in the distance between the mass centers of two clusters. We then extract the leading term of the scattering cross-section in the Born-Oppenheimer limit.
\end{abstract}

\section{Introduction}

The scattering process for multi-particle Coulomb systems with initial two-cluster data has been studied in physics litterature, both experimentally and theoretically. In particular, in the collision of a charged cluster with a neutral one ( ion-atom scattering), it is believed that if the neutral sub-system has no static dipole moment, the total cross-section would be is finite. In [ES], Enss-Simon put forward as open questions to prove the finiteness of total cross-sections in this case and to give explicit bounds for them. In [CT], Combes-Tips proved the finiteness and analyticity of forward scattering amplitude in electron-atom scattering. They indicated technical difficulties to extend their results to ion-atom collision and suggested to use Born-Oppenheimer approximation to study the problem.

Recall that it is well-known in two-body scattering theory (see, for example, [Y] ) that if the potential $V$ on $\mathbb{R}^{3}$ has the decay

$$
|V(x)| \leq C<x>^{-\rho}, \quad \forall x \in \mathbb{R}^{3}
$$

with $\rho>2$, the total cross-section for the scattering process described by $(-\Delta,-\Delta+V(x))$ is finite, while if $V(x) \sim \frac{C}{|x|^{2}}$ as $|x| \rightarrow \infty$ for some $C \neq 0$, the total cross-section is infinite. In the scattering theory for multi-particle Coulomb systems with initial two-cluster data, the intercluster interaction between the two clusters decays like $\mathcal{O}\left(|x|^{-1}\right)$ in general case, like $\mathcal{O}\left(|x|^{-2}\right.$ ) if one of the clusters is neutral ( ion-atom scattering ) and like $\mathcal{O}\left(|x|^{-3}\right)$ if the both clusters are neutral ( atom-atom scattering ). Here $x \in \mathbb{R}^{3}$ denotes the relative position of the mass-centers of the two clusters. See Appendix A for more precise statements and the calculus. For ion-atom scattering, the known results in two-body case suggest that without additional assumption, the total cross-section would be infinite. In this paper, we prove the finiteness of total crosssections under the assumption that the atom is in the fundamental state which implies, by the symmetry of Coulomb potentials, that there is no static dipole moment for the atom. The quantitive study of the total cross-sections in ion-atom scattering is interesting and non-trivial, since the leading terms in various 
known asymptotics for total cross-sections in N-body scattering ( [I1], [I2], [RW], [W]) are not defined in the present case. In this paper, we only study the asymptotics in the Born-Oppenheimer approximation, where the semiclassical parameter, $h$, is proportional to the ratio of the electonic to nuclear mass. Due to the use of cluster coordinates which is needed to describe many-particle scattering processes, the potentials become $h$-dependent. The perturbation by the shift term $l(y)=\mathcal{O}\left(h^{2}|y|\right)$ is singular and the picture of eigenvalues of the electronic Hamiltonian $P_{e}(x, h)$ defined below changes drastically from $h=0$ to $h \neq 0$. Our result in Born-Oppenheimer approximation is based on the semiclassical resolvent estimates of [KMW2] which is established in terms of the weight in $x-l(y)$, the relative position between the two nucleus. We then use the adiabatic approximation for total cross-sections and prove that the eigenvalue of the electronic Hamiltonian $P_{e}(x, h)$ converges sufficiently fast as $x \rightarrow \infty$ so that we can extract the leading term in the limit $h \rightarrow 0$.

The plan of this paper is as follows. In Section II we introduce the basic notation which will be used throughout the paper and we recall a few basic facts from N-body scattering theory. We introduce the hypotheses which are relevant for this paper and we state our main results, i.e. Theorem II.2 on the existence of the total scattering cross-section and Theorem II.3, which gives the semiclassical asymptotics of this cross-section. In Section III we prove Theorem II.2. The essential point are certain weighted $L^{2}$ estimates which show that upon localization in energy in the relevant spectral range the effective interaction decays faster than $\mathcal{O}\left(|x|^{-2}\right)$, which is the obvious norm estimate on an ion-atom interaction. In Section IV, we establish the relevant semiclassical estimates on potentials and resolvents, using methods from [KMW2] and give a sketch of the proof of Theorem II.3. In Appendix A we include the relevant expansions for the Coulomb interaction in ion-atom scattering which are used throughout the paper.

\section{Notation, assumptions and main results}

The Hamiltonian of a diatomic molecule with $N$ electrons can be written in the form

$$
\begin{aligned}
\mathrm{P}_{\text {phys }}= & \sum_{k=1}^{2} \frac{1}{2 m_{k}}\left(-\Delta_{x_{k}}\right)+\sum_{j=3}^{N+2} \frac{1}{2}\left(-\Delta_{x_{j}}\right)+\frac{Z_{1} Z_{2}}{\left|x_{1}-x_{2}\right|} \\
& +\sum_{k=1}^{2} \sum_{j=3}^{N+2} \frac{e_{j} Z_{k}}{\left|x_{j}-x_{k}\right|}+\sum_{2 \leq l<j \leq N+2} \frac{e_{l} e_{j}}{\left|x_{l}-x_{j}\right|}
\end{aligned}
$$

where $x_{k} \in \mathbb{R}^{3}, k=1,2$, denote the position of the two nuclei with mass $m_{k}$ and charge $Z_{k}>0$ and $x_{j} \in \mathbb{R}^{3}$, $j=3, \ldots, N+2$, denote the position of $N$ electrons with mass 1 and charge $e_{j} \in \mathbb{R}$ (in the physical case charges are equal and negative). Planck's constant is taken to be 1 in this formula. The result on the existence of total cross-sections remains valid for any Coulomb system.

We are interested in scattering processes where the incoming scattering channel is a two-cluster one, while the out-going scattering channel can be arbitrary. Let $a=\left(a_{1}, a_{2}\right)$ be a two-cluster decomposition of $\{1, \ldots, N+2\}$, i.e. a partition $\left(a_{1}, a_{2}\right)$ of the particle labels $\{1, \ldots, N+2\}$, where $j \in a_{j}$, for $j=1,2$. Adapted to this cluster decomposition, we choose so called clustered atomic coordinates $(x, y) \in \mathbb{R}^{3} \times \mathbb{R}^{3 N}$ :

$$
\begin{aligned}
& h=\left(\frac{1}{2 M_{1}}+\frac{1}{2 M_{2}}\right)^{1 / 2}, M_{k}=m_{k}+\left|a_{k}^{\prime}\right|, a_{k}^{\prime}=a_{k} \backslash\{k\}, k=1,2, \\
& R_{k}=\frac{1}{M_{k}}\left(m_{k} x_{k}+\sum_{j \in a_{k}^{\prime}} x_{j}\right), k=1,2, \\
& x=R_{1}-R_{2}, \\
& y_{j}=x_{j}-x_{k}, j \in a_{k}^{\prime}, k=1,2, \\
& l(y)=\frac{1}{M_{1}} \sum_{j \in a_{1}^{\prime}} y_{j}-\frac{1}{M_{2}} \sum_{j \in a_{2}^{\prime}} y_{j} .
\end{aligned}
$$

Notice that $R_{k}$ is the center of mass of the cluster $a_{k}$, for $k=1,2$, and that $x$ is the relative position of these centers of mass. These coordinates are well adapted to describe two-cluster scattering of diatomic molecules 
(see [KMW1], [KMW2]). After removing the molecular center of mass motion, the Hamiltonian $\mathrm{P}_{\text {phys }}$ may be written in this system of coordinates as

$$
\mathrm{P}=-h^{2} \Delta_{x}+\mathrm{P}_{e}(x ; h), \quad \mathrm{P}_{e}(x ; h)=\mathrm{P}^{a}(h)+\mathrm{I}_{a}(x ; h),
$$

where the sub-Hamiltonian $\mathrm{P}^{a}(h)$ is given by

$$
\mathrm{P}^{a}(h)=\mathrm{P}^{a_{1}}(h)+\mathrm{P}^{a_{2}}(h)
$$

with

$$
P^{a_{k}}(h)=\sum_{j \in a_{k}^{\prime}}\left(-\frac{1}{2} \Delta_{y_{j}}+\frac{Z_{k} e_{j}}{\left|y_{j}\right|}\right)-\frac{1}{2 m_{k}}\left(\sum_{j \in a_{k}^{\prime}} \partial_{y_{j}}\right)^{2}+\sum_{\substack{l, j \in a_{k}^{\prime} \\ l<j}} \frac{e_{l} e_{j}}{\left|y_{l}-y_{j}\right|}
$$

and the inter-cluster interaction $\mathrm{I}_{a}(x ; h)$ by

$$
\mathrm{I}_{a}(x ; h)=\frac{Z_{1} Z_{2}}{|x-l(y)|}+\sum_{\substack{k \in a_{1}^{\prime} \\ j \in a_{2}^{\prime}}} \frac{e_{k} e_{j}}{\left|y_{k}-y_{j}+x-l(y)\right|}+\sum_{j \in a_{1}^{\prime}} \frac{Z_{2} e_{j}}{\left|y_{j}+x-l(y)\right|}+\sum_{j \in a_{2}^{\prime}} \frac{Z_{1} e_{j}}{\left|x-l(y)-y_{j}\right|} .
$$

Finally, we set

$$
\mathrm{P}_{a}(h)=-h^{2} \Delta_{x}+\mathrm{P}^{a}(h) .
$$

$\mathrm{P}$ is considered as a self-adjoint operator in $L^{2}\left(\mathbb{R}^{3(N+1)} ; \mathrm{dxdy}\right)$. Note that $l(y)=\mathcal{O}\left(h^{2}|y|\right)$ and that the study of the dependence on $h$ of the spectra of $P_{e}(x ; h)$ is technical. In fact, even to prove the terme $\frac{Z_{1} Z_{2}}{|x-l(y)|}$ is uniformly ( w.r.t. $h$ ) $-\Delta_{y}$-bounded, the authors of [KMW2] used the facts that $x \in \mathbb{R}^{3}$ and $Z_{1} Z_{2}>0$.

For an arbitrary cluster decomposition $c=\left(c_{1}, \ldots, c_{k}\right)$ of $\{1, \ldots, N+2\}$, i.e. $c_{1} \cup \cdots \cup c_{k}=\{1, \ldots, N+2\}$ and $c_{j} \cap c_{k}=\emptyset$, for $j \neq k$, we can also choose adapted coordinates $\left(x_{c}, y_{c}\right)$. We call $\mathrm{P}^{c}$ the sub-Hamiltonian, $x_{c} \in \mathbb{R}^{3(k-1)}$ the inter-cluster coordinates, $y_{c}$ the intra-cluster coordinates, and $\mathrm{I}_{c}\left(x_{c}, y_{c}\right)$ the inter-cluster interaction. By $\mathrm{D}_{x_{c}}$ (resp. $\left.\mathrm{D}_{y_{c}}\right)$ and by $-\Delta_{x_{c}}$ (resp. $-\Delta_{y_{c}}$ ), we denote $-i$ times the gradient and the Laplacian in the inter-cluster (resp. intra-cluster) coordinates. It is well known (see e.g. [DG]) that, for this Schrödinger operator $\mathrm{P}$, the modified wave operators

$$
\Omega_{ \pm, \gamma}=s-\lim _{t \rightarrow \pm \infty} e^{i t \mathrm{P}} e^{-i t\left(-\Delta_{x_{c}}+\int_{0}^{t} I_{c}\left(s \mathrm{D}_{x_{c}}, 0\right) d s+E_{\gamma}\right)} J_{\gamma}
$$

exist for any scattering channel $\gamma=\left(c, E_{\gamma}, \phi_{\gamma}\right)$, where $c$ is an arbitrary cluster decomposition, $\phi_{\gamma}$ is an eigenfunction of $\mathrm{P}^{c}$ with eigenvalue $E_{\gamma}: \mathrm{P}^{c} \phi_{\gamma}=E_{\gamma} \phi_{\gamma}$, and where $J_{\gamma}$ denotes the identification operator, which is defined for any $L^{2}$-function $f$ of the variable $x_{c}$ by

$$
\left(J_{\gamma} f\right)\left(x_{c}, y_{c}\right)=f\left(x_{c}\right) \phi_{\gamma}\left(y_{c}\right)
$$

Furthermore, the family of wave operators $\left\{\Omega_{ \pm, \gamma}, \forall \gamma\right\}$ is asymptotically complete. It is equally well known (see [Ra]) that, if $a=\left(a_{1}, a_{2}\right)$ is a two-cluster decomposition with one neutral cluster (an atom), say $a_{1}$, i.e.

$$
\sum_{j \in a_{1}^{\prime}} e_{j}=-Z_{1}
$$

then, for any channel $\alpha=\left(a, E_{\alpha}, \phi_{\alpha}\right)$ with $E_{\alpha}$ outside the thresholds of $\mathrm{P}^{a}$, one can define the wave operators without modifier, namely by

$$
\Omega_{ \pm, \alpha}^{\prime}=s-\lim _{t \rightarrow \pm \infty} e^{i t \mathrm{P}} e^{-i t\left(-\Delta_{x_{a}}+E_{\alpha}\right)} J_{\alpha}
$$

In this case, $\Omega_{ \pm, \alpha}=\Omega_{ \pm, \alpha}^{\prime} e^{i \psi\left(D_{x_{a}}\right)}$, where $\psi$ is a real function. Therefore the result on asymptotic completeness remains true if we replace $\Omega_{ \pm, \alpha}$ by $\Omega_{ \pm, \alpha}^{\prime}$ when the latter exists. So we just set $\Omega_{ \pm, \gamma}=\Omega_{ \pm, \gamma}^{\prime}$ if they 
exist. For any two scattering channels $\gamma, \beta$, we then define the associated scattering operator from channel $\gamma$ to channel $\delta$ by

$$
\mathrm{S}_{\beta \gamma}=\Omega_{+, \beta}^{*} \Omega_{-, \gamma}, \quad \mathrm{T}_{\delta \gamma}=\mathrm{S}_{\beta \gamma}-\delta_{\beta \gamma},
$$

where $\delta_{\beta \gamma}=1$ if $\gamma=\beta$ and 0 otherwise.

Let us now define the total scattering cross-sections in many-particle scattering. Since few is known about the scattering amplitudes in many-body scattering theory (see [V] for results in this subject), we define the total scattering cross-sections according to the philosophy of [ES]. For $\lambda \geq E_{\alpha}(h)$, we introduce the magnitude of the momentum associated with the kinetic energy of the relative motion of the two clusters in the scattering channel $\alpha$ via

$$
n_{\alpha}(\lambda ; h):=\lambda_{\alpha}^{1 / 2}(h), \quad \lambda_{\alpha}(h):=\lambda-E_{\alpha}(h) .
$$

For $\left.g \in C_{0}^{\infty}\left(I_{\alpha} ; \mathbb{C}\right), I_{\alpha}=\right] E_{\alpha}(h) ;+\infty\left[\right.$, and $\omega \in \mathbb{S}^{2}$, we consider the wave packet

$$
\mathbb{R}^{3} \ni x \mapsto g_{\omega}(x)=\tilde{g}(\omega \cdot x)
$$

where

$$
\tilde{g}(\nu)=\frac{1}{2 \sqrt{\pi h}} \int_{\mathbb{R}} e^{i h^{-1} n_{\alpha}(\mu ; h) \nu} \frac{g(\mu)}{n_{\alpha}(\mu ; h)^{1 / 2}} d \mu .
$$

The normalization is chosen such that

$$
\|g\|_{L^{2}(\mathbb{R})}=\|\tilde{g}\|_{L^{2}(\mathbb{R})} .
$$

Denoting by $\mathcal{C}$ the set of all channels, we want to apply, for $\delta \in \mathcal{C}, \mathrm{T}_{\delta \alpha}$ to $g_{\omega}(x) \phi_{\alpha}(y ; h)$. Since this function does not belong to $L^{2}\left(\mathbb{R}^{3(N+1)}\right)$ - it decays rapidly only in the direction defined by $\omega$ - we regularize it by multiplication with a function $h_{R, \omega} \in L^{\infty}\left(\mathbb{R}^{3}\right)$, depending only on the variable $x-(\omega \cdot x) \omega$ transversal to the direction $\omega$ of the incident wave packet $g_{\omega}(x)$, such that pointwisely

$$
\lim _{R \rightarrow \infty} h_{R, \omega}=1 .
$$

For the purpose of this paper we shall specify this cut-off function to be a Gaussian, i.e. we take

$$
h_{R, \omega}(x)=e^{-(x-(\omega \cdot x) \omega)^{2} / R}
$$

Definition. For $\lambda \in I_{\alpha}$ and $\omega \in \mathbb{S}^{2}$, we shall say that the total cross-section $\sigma_{\alpha}(\lambda, \omega)$ with the incoming channel $\alpha$ exists at the energy $\lambda$ with the incident direction $\omega$, if the following limit is finite and well defined:

$$
\sigma_{\alpha}(\lambda, \omega):=\lim _{n \rightarrow \infty} \lim _{R \rightarrow \infty} \sum_{\delta \in \mathcal{C}}\left\|\mathrm{T}_{\delta \alpha} h_{R, \omega} g_{n, \omega} \phi_{\alpha}\right\|^{2},
$$

where $g_{n, \omega}$ is defined as in (II.16) with $g$ replaced by $g_{n}$ :

$$
g_{n}(\mu)=n^{-1 / 2} h((\mu-\lambda) / n)
$$

and $h$ is any $C_{0}^{\infty}(\mathbb{R})$-function normalized by $\int_{\mathbb{R}}|h(\mu)|^{2} d \mu=1$.

Recall that in [ES] and [W], the total cross-section is defined as distribution in $\mu \in I_{\alpha}$ by

$$
\int_{E_{\alpha}(h)}^{+\infty} \sigma_{\alpha}(\mu, \omega)|g(\mu)|^{2} d \mu=\lim _{R \rightarrow \infty} \sum_{\delta \in \mathcal{C}}\left\|\mathrm{T}_{\delta \alpha} h_{R, \omega} g_{\omega} \phi_{\alpha}\right\|^{2},
$$

for all $g \in C_{0}^{\infty}\left(I_{\alpha} ; \mathbb{C}\right)$. Since $\left|g_{n}(\cdot)\right|^{2}$ converges to $\delta_{\lambda}(\cdot)$, the Dirac measure at $\lambda$, as $n \rightarrow \infty$, the definitions (II.19) and (II.20) coincide if the distribution defined in (II.20) can be identified with a continuous function in a neighbourhood of $\lambda$, which is true in the case when one knows to prove the existence in the sense of 
distributions ( see $[\mathrm{RW}, \mathrm{W}]$ ). For physical background of this definition and its equivalence to the usual one in two-body case, see [ES], [RW], [W], [Jec]. For some channels $\gamma, \delta$ and some incident direction $\omega$, total scattering cross-sections may not exist on any interval $I$ (see $[\mathrm{W}]$ ). Usually it is required that the interactions decay quite rapidly to ensure their existence. In the present situation with Coulomb interactions, which a priori do not decay sufficiently fast, we shall show the existence, i.e. finiteness, of $\sigma_{\alpha}$ only for some special channel $\alpha$ describing ion-atom scattering, for all incident directions $\omega \in \mathbb{S}^{2}$. The conditions on $\alpha$ are collected in the following hypothesis.

Hypothesis 1 . Let $\alpha=\left(a, E_{\alpha}, \phi_{\alpha}\right)$ be a channel with $E_{\alpha} \in \sigma_{\mathrm{disc}}\left(\mathrm{P}^{a}\right)$ and cluster decomposition a $=\left(a_{1}, a_{2}\right)$ such that each cluster contains a nucleus and such that $a_{1}$ is neutral (an atom), that is

$$
\sum_{j \in a_{1}^{\prime}} e_{j}+Z_{1}=0 .
$$

Assume that

$$
E_{\alpha}=E_{\alpha, 1}+E_{\alpha, 2} \quad \text { with } E_{\alpha, j} \in \sigma_{\mathrm{disc}}\left(\mathrm{P}^{a_{j}}\right), j=1,2,
$$

where $\mathrm{P}^{a_{j}}$ stands for the internal Hamiltonian of cluster $a_{j}$ and $E_{\alpha, 1}$ (the eigenvalue of the neutral cluster) is non-degenerate.

Remark II.1. Write $y=\left(y^{\prime}, y^{\prime \prime}\right)$ for the electronic coordinates in the clusters $a_{1}, a_{2}$ and put

$$
\begin{aligned}
\phi_{\alpha}(y) & =\phi_{\alpha, 1}\left(y^{\prime}\right) \phi_{\alpha, 2}\left(y^{\prime \prime}\right), \quad \forall y \in \mathbb{R}^{3 N}, \\
\text { with } \mathrm{P}^{a_{j}} \phi_{\alpha, j} & =E_{\alpha, j} \phi_{\alpha, j} .
\end{aligned}
$$

By the spherical symmetry of Coulomb potential and the non-degeneracy of $E_{\alpha, 1}$, it can be deduced that $\left|\phi_{\alpha, 1}\left(-y^{\prime}\right)\right|=\left|\phi_{\alpha, 1}\left(y^{\prime}\right)\right|$. Therefore,

$$
\int_{\mathbb{R}^{3\left|a_{1}^{\prime}\right|}} y_{j}\left|\phi_{\alpha, 1}\left(y^{\prime}\right)\right|^{2} d y^{\prime}=0, \quad \forall j \in a_{1}^{\prime} .
$$

Since $a_{1}$ is neutral, an elementary calculus using the Taylor expansion of $I_{a}$ in $y$ shows that

$$
<I_{a}(x, h) \phi_{\alpha}, \phi_{\alpha}>_{y}=\mathcal{O}\left(|x|^{-3}\right) \text {. }
$$

$<\cdot, \cdot>_{y}$ denotes the scalar product in $L^{2}\left(\mathbb{R}_{y}^{3 N} ; d y\right)$.

We denote by $\mathrm{R}(z ; h)$ the resolvent of $\mathrm{P}(h)$ and recall that its boundary value $\mathrm{R}(\lambda \pm i 0 ; h): L^{2, s} \rightarrow L^{2,-s}$ is well defined outside the set $\mathcal{T}$ of the thresholds and the eigenvalues of $\mathrm{P}(h)$ as an operator between the weighted $L^{2}$ spaces, for any $s>1 / 2$.

Our first main result concerns the existence of $\sigma_{\alpha}$ and gives a useful formula for it.

Theorem II.2. Let $\alpha=\left(a, E_{\alpha}(h), \phi_{\alpha}(h)\right)$ be a scattering channel satisfying Hypothesis 1 . We set

$$
F(z, \omega ; h)=\left\langle\mathrm{R}(z, h) \mathrm{I}_{a} e_{\alpha}, \mathrm{I}_{a} e_{\alpha}\right\rangle, \quad \operatorname{Im} z \neq 0,
$$

where

$$
e_{\alpha}(x, y)=e^{i h^{-1} n_{\alpha}(\lambda ; h) \omega \cdot x} \phi_{\alpha}(y ; h) .
$$

Let $\mathcal{T}$ be the set of thresholds and eigenvalues of $\mathrm{P}$. Then, for any energy $\lambda \in I_{\alpha} \backslash \mathcal{T}$ and any incident direction $\omega \in \mathbb{S}^{2}$, the limit

$$
F(\lambda+i 0, \omega ; h)=\lim _{\epsilon \rightarrow 0_{+}} F(\lambda+i \epsilon, \omega ; h)
$$

exists and defines a continuous function in $\lambda$. The total scattering cross-section $\sigma_{\alpha}(\lambda, \omega)$ exists for any energy $\lambda \in I_{\alpha} \backslash \mathcal{T}$ and any incident direction $\omega \in \mathbb{S}^{2}$ and one has the optical formula

$$
\sigma_{\alpha}(\lambda, \omega)=\frac{1}{h n_{\alpha}(\lambda ; h)} \operatorname{Im} F(\lambda+i 0, \omega ; h) .
$$


Since $\mathrm{I}_{a} e_{\alpha}$ does not belong to $L^{2, s}$, for some $s>1 / 2$, this result is not trivial. Its proof - given in Section III - depends crucially on the decay of some appropriate effective potentials, combined with phase space analysis, i.e. an appropriate localization in the relative kinetic energy of the two clusters.

Next we are interested in the Born-Oppenheimer approximation $(h \rightarrow 0)$ of $\sigma_{\alpha}$. We restrict ourselves to the groundstate energy of $\mathrm{P}^{a}$ and demand some stability property w.r.t. $x$ and $h$.

Hypothesis 2. Let $h_{0}>0$ be small enough. Let $E_{\alpha}(h)$, satisfying Hypthesis 1 , be the bottom of the spectrum of $\mathrm{P}^{a}(h), 0 \leq h \leq h_{0}$. Let $\lambda_{0}>E_{\alpha}(0)$. From (II.7), we see that, for some $\delta>0, \lambda_{0}-\delta>E_{\alpha}(h), 0<h \leq h_{0}$. Let $\lambda_{1}(x ; h)$ be the bottom of the spectrum of $\mathrm{P}_{e}(x ; h)$. We assume that for $x$ in a neighborhood $\mathcal{O}_{\lambda_{0}}$ of the non-compact set

$$
\left\{x \in \mathbb{R}^{3} ; \lambda_{1}(x ; 0) \leq \lambda_{0}\right\},
$$

$\lambda_{1}(x ; h)$ is a simple eigenvalue and is the unique eigenvalue of $\mathrm{P}_{e}(x ; h)$ that tends to $E_{\alpha}(h)$ as $|x| \rightarrow \infty$, and the unique eigenvalue of $\mathrm{P}_{e}(x ; h)$ that tends to $\lambda_{1}(x ; 0)$ as $h \rightarrow 0$. Furthermore, we demand that

$$
\begin{aligned}
& \lambda_{1}(x ; h) \rightarrow E_{\alpha}(h) \quad \text { as } \quad|x| \rightarrow \infty \text {, uniformly w.r.t. } h \leq h_{0}, \\
& \lambda_{1}(x ; h) \rightarrow \lambda_{1}(x ; 0) \quad \text { as } \quad h \rightarrow 0 \text {, uniformly w.r.t. } x \in \mathcal{O}_{\lambda_{0}} \text {. }
\end{aligned}
$$

Note that there exists $\delta_{0}>0$, such that, for $h_{0}$ small enough and $0 \leq h \leq h_{0}$,

$$
\left\{x \in \mathbb{R}^{3} ; \lambda_{1}(x ; h) \leq \lambda_{0}+\delta_{0}\right\} \subset \mathcal{O}_{\lambda_{0}} .
$$

We also impose that for $0 \leq h \leq h_{0}$,

$$
\inf _{x \in \mathcal{O}_{\lambda_{0}}}\left(\sigma\left(\mathrm{P}_{e}(x ; h)\right) \backslash\left\{\lambda_{1}(x ; h)\right\}\right)>\lambda_{0}+2 \delta_{0},
$$

where $\sigma\left(\mathrm{P}_{e}(x ; h)\right)$ denotes the spectrum of $\mathrm{P}_{e}(x ; h)$.

For $x \in \mathcal{O}_{\lambda_{0}}$, let $\psi_{e}(x ; h)$ be a normalized eigenfunction of $\mathrm{P}_{e}(x ; h)$ associated to $\lambda_{1}(x ; h)$. As in [KMW2], we can extend it to a smooth, normalized function $\phi_{e}(x ; h)$ of $x$ such that, for some $\delta_{1}>0$,

$$
\left\langle\mathrm{P}_{e}(x ; h) \phi_{e}(x ; h), \phi_{e}(x ; h)\right\rangle \geq \lambda_{0}+\delta_{1},
$$

for all $0 \leq h \leq h_{0}$ and for all $x$ in some compact neighborhood $K$ of the complement of $\mathcal{O}_{\lambda_{0}}$, satisfying

$$
K \subset\left\{x \in \mathbb{R}^{3} ; \lambda_{1}(x ; h)>\lambda_{0}, 0 \leq h \leq h_{0}\right\} .
$$

We denote the orthogonal projection on the one-dimensional space generated by $\phi_{e}(x ; h)$ in $L^{2}\left(\mathbb{R}_{y}^{3 N}\right)$ by $\Pi(x, h)$. It induces a projection $\Pi(h)$ on $L^{2}\left(\mathbb{R}^{3(N+1)}\right)$. The orthogonal projection $\Pi_{0}(h)$ onto $\phi_{\alpha}(h)$ (introduced in Hypothesis 1$)$ also induces a projection on $L^{2}\left(\mathbb{R}^{3(N+1)}\right)$, which we still denote by $\Pi_{0}(h)$. We then define the adiabatic operator associated with the spectral projection $\Pi(h)$ by

$$
\mathrm{P}^{A D}(h):=\Pi(h) \mathrm{P} \Pi(h) .
$$

We denote by $\mathrm{R}^{A D}(z ; h)$ its resolvent and set $\hat{\Pi}(h)=1-\Pi(h)$ and $\hat{\Pi}_{0}(h)=1-\Pi_{0}(h)$.

We consider an energy range $J \subset] E_{\alpha}(0) ; \lambda_{0}\left[\right.$. Let $\psi_{t}$ be the Hamiltonian flow of the effective Hamiltonian function

$$
\mathrm{H}_{\mathrm{eff}}(x, \xi)=|\xi|^{2}+\lambda_{1}(x ; 0)-E_{\alpha}(0) .
$$

An energy $\lambda \in \mathbb{R}$ is non-trapping for $\mathrm{H}_{\mathrm{eff}}$ if, for all $(x, \xi)$ belonging to the energy surface of $\mathrm{H}_{\mathrm{eff}}$ of energy $\lambda$, the point $\psi_{t}(x, \xi)$ goes to infinity as $t$ and $-t$ go to $+\infty$.

Hypothesis 3. Let $J$ an open interval of $\mathbb{R}$ such that $J$ is non-trapping for the effective Hamiltonian function $\mathrm{H}_{\mathrm{eff}}$, i.e. $\lambda$ is a non-trapping energy for $\mathrm{H}_{\mathrm{eff}}$ for all $\lambda \in J$. 
Note that such an interval $J$ is contained in $I_{\alpha} \backslash \mathcal{T}$, for $h$ small enough. Thus Theorem II.2 holds on $J$. In our context we need such a hypothesis to obtain a semiclassical estimate on the resolvent.

Under the previous hypotheses, we shall derive in Proposition IV.1 semiclassical estimates on $\mathrm{R}(\lambda \pm i 0)$ and $\mathrm{R}^{\mathrm{AD}}(\lambda \pm i 0)$, for $\lambda \in J$, using arguments developed in [KMW2]. Finally we introduce the effective potentials which govern the leading terms of $\sigma_{\alpha}$. Denoting by $C_{2}$ the electronic charge of $a_{2}$, that is

$$
C_{2}=\sum_{j \in a_{2}^{\prime}} e_{j}
$$

we define the function

$$
C(\hat{x}, y)=\left(C_{2}+Z_{2}\right) \sum_{l \in a_{1}^{\prime}} e_{l} \hat{x} \cdot y_{l}
$$

where $\hat{x}=x /|x|$ and where $\cdot$ denotes the standard scalar product in $\mathbb{R}^{3}$. Physically, this function describes the interaction of the dipoles formed by the electrons in cluster $a_{1}$ with the effective charge of cluster $a_{2}$. Define

$$
\hat{R}_{a}(h)=\left(P^{a}(h) \hat{\Pi}_{0}(h)-E_{\alpha}(h)\right)^{-1} \hat{\Pi}_{0}(h) .
$$

The effective potential in the context of the Born-Oppenheimer approximation is given by

$$
\mathrm{I}_{\mathrm{eff}}(x):=\lambda_{1}(x ; 0)-E_{\alpha}(0) .
$$

While the intuitive effective term $<I_{a}(x ; 0) \phi_{\alpha}(0), \phi_{a}(0)>_{y}$ may decay exponentially, we shall prove that $\mathrm{I}_{\text {eff }}(x)$ is exactly of the order $\mathcal{O}\left(|x|^{-4}\right)$. In fact, we prove in Lemma IV.2 that

$$
\left|\mathrm{I}_{\text {eff }}(x)-\hat{\mathrm{I}}_{\text {eff }}(x)\right|=\mathcal{O}\left(|x|^{-5}\right), \quad \text { as }|x| \rightarrow \infty
$$

where

$$
\hat{\mathrm{I}}_{\mathrm{eff}}(x):=-2\left\langle\hat{R}_{a}(0) \hat{\Pi}_{0}(0) C(\hat{x}, y) \phi_{\alpha}(0), \hat{\Pi}_{0}(0) C(\hat{x}, y) \phi_{\alpha}(0)\right\rangle_{L^{2}\left(\mathbb{R}_{y}^{3 N}\right)}|x|^{-4}
$$

is everywhere negative if $C_{2}+Z_{2} \neq 0$. It is essentially this fact which allows to extract the leading order of the total scattering cross-section in equation (II.39) below. Now we can state our second main result, which gives the semiclassical asymptotics of $\sigma_{\alpha}$.

Theorem II.3. Let $\alpha=\left(a, E_{\alpha}(h), \phi_{\alpha}(h)\right)$ be a scattering channel satisfying Hypothesis 1 and Hypothesis 2. Let $J$ be a real interval satisfying Hypothesis 3. Then we have

$$
\sigma_{\alpha}(\lambda, \omega)=O\left(h^{-2 / 3}\right)
$$

locally uniformly w.r.t. $\lambda \in J$ and $\omega \in \mathbb{S}^{2}$. We set $n_{\alpha}(\lambda ; 0)=\left(\lambda-E_{\alpha}(0)\right)^{1 / 2}$ and we denote by $H_{\omega}$ the hyperplane orthogonal to $\omega$. Then there exists some $\epsilon_{0}>0$ such that, for either choice of effective potential, i.e. for $\mathrm{I}=\mathrm{I}_{\mathrm{eff}}$ and $\mathrm{I}=\hat{\mathrm{I}}_{\mathrm{eff}}$, we have

$$
\sigma_{\alpha}(\lambda, \omega)=4 \int_{H_{\omega}} \sin ^{2}\left(\frac{1}{4 h n_{\alpha}(\lambda ; 0)} \int_{\mathbb{R}} \mathrm{I}(u+s \omega) d s\right) d u+O\left(h^{-2 / 3+\epsilon_{0}}\right)
$$

locally uniformly w.r.t. $\lambda \in J$ and $\omega \in \mathbb{S}^{2}$. If $a_{2}$ is not neutral (i.e. the electronic charge $C_{2}$ of $a_{2}$ satisfies $C_{2} \neq-Z_{2}$ ), the leading term (II.39) with $\mathrm{I}=\hat{\mathrm{I}}_{\text {eff }}$ is exactly of order $h^{-2 / 3}$ and thus is $\sigma_{\alpha}$.

Theorem II.3 shows that the Born-Oppenheimer approximation correctly describes the asymptotics of the total scattering cross-section in the situation considered in this paper, as expected in [CT]. 


\section{Existence of the total scattering cross-section}

In this section we shall prove the existence of the total scattering cross-section as stated in Theorem II.2. The parameter $h$ plays no role in this section and will be set to 1 . We shall assume throughout this section that the initial channel $\alpha$ is associated to a two-cluster decomposition $a=\left(a_{1}, a_{2}\right)$ with $a_{1}$ a neutral cluster, that is, (II.21) holds for $a_{1}$. As a first step, we establish the following representation formula. Here we use the function $u_{R, \omega}=g_{\omega} h_{R, \omega}$, where $g_{\omega}, h_{R, \omega}$ are defined in (II.16) and (II.18).

Lemma III.1. For $\left.g \in C_{0}^{\infty}\left(I_{\alpha} ; \mathbb{C}\right), I_{\alpha}:=\right] E_{\alpha} ;+\infty[$, one has

$$
\sum_{\beta \in \mathcal{C}}\left\|T_{\beta \alpha} u_{R, \omega} \phi_{\alpha}\right\|^{2}=4 \pi \int_{I_{\alpha}} \operatorname{Im}\left\langle R(\lambda+i 0) \mathrm{I}_{a} \phi_{\alpha} u_{R, \omega}(\lambda), \mathrm{I}_{a} \phi_{\alpha} u_{R, \omega}(\lambda)\right\rangle d \lambda
$$

where

$$
u_{R, \omega}(\lambda, x)=\frac{R}{8}\left(\frac{n_{\alpha}(\lambda)}{\pi}\right)^{3 / 2} \int_{\mathbb{S}_{+}^{2}} e^{i n_{\alpha}(\lambda) x \cdot \theta-\frac{R}{4} \lambda_{\alpha}\left(\theta_{2}^{2}+\theta_{3}^{2}\right)} \sqrt{\theta_{1}} g\left(\lambda_{\alpha} \theta_{1}^{2}+E_{\alpha}\right) d \theta
$$

where $\theta_{1}=\theta \cdot \omega$, the components $\theta_{2}, \theta_{3}$ denote the directions orthogonal to $\omega \in \mathbb{S}^{2}$ and $\mathbb{S}_{+}^{2}$ denotes the half sphere $\theta_{1}>0, \theta \in \mathbb{S}^{2}$.

The proof is the same as in [RW, W] and is omitted here. Remark that the asymptotic completeness of wave operators plays an essential role in the proof.

Writing $\theta^{\prime}=\left(\theta_{2}, \theta_{3}\right)$, setting $B_{\epsilon, R}=\left\{\theta^{\prime} \in \mathbb{R}^{2} ;\left|\theta^{\prime}\right| \leq R^{-(1-\epsilon) / 2}\right\}$ and using $d \theta=\left(1-{\theta^{\prime}}^{2}\right)^{-1 / 2} d \theta^{\prime}$ on $\mathbb{S}_{+}^{2}$, we note that equation (III.2) implies

$$
\begin{array}{r}
u_{R, \omega}(\lambda, x)=\frac{R}{8}\left(\frac{n_{\alpha}(\lambda)}{\pi}\right)^{3 / 2} \int_{B_{\epsilon, R}} e^{i n_{\alpha}(\lambda)\left(x_{1} \sqrt{1-\theta^{\prime 2}}+x^{\prime} \cdot \theta^{\prime}\right)} e^{-\frac{R}{4} \lambda_{\alpha} \theta^{\prime 2}}\left(1-\theta^{\prime 2}\right)^{-1 / 4} g\left(\lambda-\lambda_{\alpha} \theta^{\prime 2}\right) d \theta^{\prime} \\
+\mathcal{O}_{\epsilon}\left(\left|R \lambda_{\alpha}\right|^{-\infty}\right)
\end{array}
$$

uniformly in $x \in \mathbb{R}^{3}$. For $\left|\theta^{\prime}\right| \leq R^{-(1-\epsilon) / 2}$ we change variables via $\tau=\sqrt{R} \theta^{\prime}$ and, considering separately the regions $|x|>R^{\epsilon / 2}$ and $|x|<R^{\epsilon / 2}$, we observe that, for $\epsilon$ sufficiently small,

$$
\langle x\rangle^{-\epsilon}\left|e^{i n_{\alpha}(\lambda)\left(x_{1} \sqrt{1-\tau^{2} / R}+\frac{\tau}{\sqrt{R}} \cdot x^{\prime}\right)}-e^{i n_{\alpha}(\lambda) x_{1}}\right| \leq C n_{\alpha}(\lambda)\left(1+\tau^{2}\right) R^{-\epsilon / 2} .
$$

Taylor expansion of the integrand in equation (III.3) combined with the evaluation of the Gaussian integral

$$
\int_{\mathbb{R}^{2}} e^{-\frac{R}{4} \lambda_{\alpha} \theta^{\prime 2}} d \theta^{\prime}=\frac{4 \pi}{R \lambda_{\alpha}}
$$

gives

Lemma III.2. For any $\epsilon>0, N \in \mathbb{N}$ there exists $C>0$ such that

$$
\left|u_{R, \omega}(x, \lambda)-\frac{1}{2}\left(\frac{1}{\pi n_{\alpha}(\lambda)}\right)^{1 / 2} g(\lambda) e^{i n_{\alpha}(\lambda) x \cdot \omega}\right| \leq C\langle x\rangle^{\epsilon} R^{-\epsilon / 2}\left|n_{\alpha}(\lambda)\right|^{-N}
$$

uniformly in $x \in \mathbb{R}^{3}, R \geq 1$ and $n_{\alpha}(\lambda) \geq c>0$.

We shall now derive Theorem II.2 as an easy consequence of

Theorem III.3. Let $\chi \in C_{0}^{\infty}(\mathbb{R})$ be equal to 1 on $[-\delta / 2, \delta / 2]$ with supp $\chi \subset(-\delta, \delta)$. Assuming Hypothesis 1 , there exists $\delta>0$ such that for any $\lambda \in I_{\alpha} \backslash \mathcal{T}$ and for $u, v \in L^{\infty}\left(\mathbb{R}_{x}^{3}\right)$ with

$$
\chi\left(-\Delta_{x}-\lambda_{\alpha}\right) u=u, \quad \chi\left(-\Delta_{x}-\lambda_{\alpha}\right) v=v
$$

one has

$$
\left|\left\langle R(\lambda+i \epsilon) \mathrm{I}_{a} \phi_{\alpha} u, \mathrm{I}_{a} \phi_{\alpha} v\right\rangle\right| \leq C_{s}\left\|\langle x\rangle^{-s} u\right\|_{L^{\infty}}\left\|\langle x\rangle^{-s} v\right\|_{L^{\infty}}
$$


where $0 \leq s<1 / 2$ and $C_{s}$ is independent of $\lambda$ in any compact subset of $I_{\alpha} \backslash \mathcal{T}$ and $\left.\left.\epsilon \in\right] 0,1\right]$. The weak limit

$$
\left\langle R(\lambda+i 0) \mathrm{I}_{a} \phi_{\alpha} u, \mathrm{I}_{a} \phi_{\alpha} v\right\rangle:=\lim _{\epsilon \rightarrow 0_{+}}\left\langle R(\lambda+i \epsilon) \mathrm{I}_{a} \phi_{\alpha} u, \mathrm{I}_{a} \phi_{\alpha} v\right\rangle
$$

exists and defines a continuous function of $\lambda$ in $I_{\alpha} \backslash \mathcal{T}$.

Proof of Theorem II.2: It is well known that the map

$$
\left(I_{\alpha} \backslash \mathcal{T}\right) \ni \lambda \mapsto\langle(x, y)\rangle^{-s} R(\lambda+i 0)\langle(x, y)\rangle^{-s}
$$

is continuous for any $s>1 / 2$. From Theorem III.3, we see that the function

$$
F(\lambda+i 0, \omega):=\left\langle R(\lambda+i 0) \mathrm{I}_{a} \phi_{\alpha} e^{i n_{\alpha}(\lambda) x \cdot \omega}, \mathrm{I}_{a} \phi_{\alpha} e^{i n_{\alpha}(\lambda) x \cdot \omega}\right\rangle
$$

is well defined and continuous for $\lambda \in I_{\alpha} \backslash \mathcal{T}$. Let $u_{R, \omega}(\lambda)$ be the function defined in Lemma III.1. Then $u_{R, \omega}(\lambda)$ and $e^{i n_{\alpha}(\lambda) x \cdot \omega}$ are $L^{\infty}$-functions satisfying the condition (III.5) in Theorem III.3. Therefore, combining Lemma III. 2 with the definition of $f_{\alpha}$ and $u_{R, \omega}(\lambda)$, we find that for some $0<s<1 / 2$

$$
\begin{aligned}
& \left|\left\langle R(\lambda+i 0) \mathrm{I}_{a} \phi_{\alpha} u_{R, \omega}(\lambda), \mathrm{I}_{a} \phi_{\alpha} u_{R, \omega}(\lambda)\right\rangle-\frac{|g(\lambda)|^{2}}{4 \pi n_{\alpha}(\lambda)} F(\lambda, \omega)\right| \\
& \leq C\left\|\langle x\rangle^{-s}\left(u_{R, \omega}(\lambda)-\frac{g(\lambda)}{2\left(\pi n_{\alpha}(\lambda)\right)^{1 / 2}} e^{i n_{\alpha}(\lambda) x \cdot \omega}\right)\right\|_{L^{\infty}} \\
& \quad \leq C_{M} R^{-s / 2}\left|n_{\alpha}(\lambda)\right|^{-M},
\end{aligned}
$$

for all $M,\left|n_{\alpha}(\lambda)\right| \geq c>0$. This estimate proves that for any $g \in C_{0}^{\infty}\left(I_{\alpha} \backslash \mathcal{T}\right)$, the limit

$$
\lim _{R \rightarrow \infty} \sum_{\beta \in \mathcal{C}}\left\|T_{\beta \alpha} h_{R, \omega} g_{\omega} \phi_{\alpha}\right\|^{2}
$$

exists and is equal to

$$
\int \operatorname{Im} F(\lambda+i 0, \omega) \frac{|g(\lambda)|^{2}}{n_{\alpha}(\lambda)} d \lambda
$$

Now we replace $g$ by $g_{n}$ in the above formula and take the limit $n \rightarrow \infty$. Since $F(\lambda+i 0, \omega)$ is continuous in $\lambda \in I_{\alpha} \backslash \mathcal{T}$, we obtain from the definition of total cross section that $\sigma_{\alpha}(\lambda, \omega)$ exists and

$$
\sigma_{\alpha}(\lambda, \omega)=\frac{1}{n_{\alpha}(\lambda)} \operatorname{Im} F(\lambda+i 0, \omega)
$$

for $\lambda \in I_{\alpha} \backslash \mathcal{T}$ and $\omega \in \mathbb{S}^{2}$.

The remaining part of this section is devoted to proving Theorem III.3. This is divided into several steps which shall be stated as distinct Lemmata. Here we are inspired by the weighted $L^{2}$ estimates and the phase space decomposition in $[\mathrm{CT}]$.

Lemma III.4. If $u \in L^{\infty}\left(\mathbb{R}_{x}^{3}\right)$ satisfies $\chi\left(-\Delta_{x}-\lambda_{\alpha}\right) u=u$, with $\chi$ as in Theorem III.3, then

$$
\left(1-\chi\left(-\Delta_{x}-\lambda_{\alpha}\right)\right) \mathrm{I}_{a} \phi_{\alpha} u \in L^{2, s}\left(\mathbb{R}^{3(N+1)}\right)
$$

for any $s<3 / 2$ and

$$
\left\|\left(1-\chi\left(-\Delta_{x}-\lambda_{\alpha}\right)\right) \mathrm{I}_{a} \phi_{\alpha} u\right\|_{L^{2, s}\left(\mathbb{R}^{3(N+1)}\right)} \leq C_{s, s^{\prime}}\left\|\langle x\rangle^{-s^{\prime}} u\right\|_{L^{\infty}}
$$

for any $s, s^{\prime}$ with $s+s^{\prime}<3 / 2$. 
Proof: Let $\Gamma$ be the set of all possible collisions between nuclei and electrons, described in the coordinates $(x, y)$. We choose a cut-off function $\tilde{\chi} \in C_{0}^{\infty}\left(\mathbb{R}^{3(N+1)}\right)$ with $0 \leq \tilde{\chi} \leq 1$, which is equal to 1 in a small conic neighborhood of $\Gamma$ and vanishes outside a slightly bigger conic neighborhood. Then

$$
\tilde{\chi} \mathrm{I}_{a} \phi_{\alpha} u \in L^{2, s}\left(\mathbb{R}^{3(N+1)}\right) \quad \text { and }\left(1-\chi\left(-\Delta_{x}-\lambda_{\alpha}\right)\right) \tilde{\chi} \mathrm{I}_{a} \phi_{\alpha} u \in L^{2, s}\left(\mathbb{R}^{3(N+1)}\right), \quad \forall s>0
$$

On the support of $1-\tilde{\chi}$, the interaction potential $\mathrm{I}_{a}$ is smooth, and since the cluster $a_{1}$ is neutral, we have for $\tilde{\mathrm{I}}_{a}=(1-\tilde{\chi}) \mathrm{I}_{a}$

$$
\tilde{\mathrm{I}}_{a}(x, y) \phi_{\alpha}=\mathcal{O}\left(|x|^{-2}\right), \quad \partial_{x} \tilde{\mathrm{I}}_{a}(x, y) \phi_{\alpha}=\mathcal{O}\left(|x|^{-3}\right) \quad \text { in } L^{2, s}\left(\mathbb{R}_{y}^{3 N}\right), \forall s>0 .
$$

Next we rewrite

$$
\left(1-\chi\left(-\Delta_{x}-\lambda_{\alpha}\right)\right)\left(\tilde{\mathrm{I}}_{a} \phi_{\alpha} u\right)=-\left[\chi\left(-\Delta_{x}-\lambda_{\alpha}\right), \tilde{\mathrm{I}}_{a}\right]\left(\phi_{\alpha} u\right)
$$

The kernel of the commutator $-\left[\chi\left(-\Delta_{x}-\lambda_{\alpha}\right), \tilde{I}_{a}\right]$ is given by

$$
\begin{aligned}
K\left(x, x^{\prime}\right) & =\frac{1}{(2 \pi)^{3}} \int\left(\tilde{\mathrm{I}}_{a}(x, y)-\tilde{\mathrm{I}}_{a}\left(x^{\prime}, y\right)\right) e^{i \xi \cdot\left(x-x^{\prime}\right)} \chi\left(\xi^{2}-\lambda_{\alpha}\right) d \xi \\
& =\frac{i}{(2 \pi)^{3}} \int e^{i h^{-1} \xi \cdot\left(x-x^{\prime}\right)} \int_{0}^{1}\left(2 \xi \cdot \partial_{x} \tilde{\mathrm{I}}_{a}\right)\left(x^{\prime}+t\left(x-x^{\prime}\right), y\right) d t \chi^{\prime}\left(\xi^{2}-\lambda_{\alpha}\right) d \xi
\end{aligned}
$$

An easy analysis shows that

$$
\left[\chi\left(-\Delta_{x}-\lambda_{\alpha}\right), \tilde{\mathrm{I}}_{a}\right]\left(\phi_{\alpha} u\right)=\mathcal{O}\left(|x|^{-3}\right) \quad \text { in } \quad L^{2, s}\left(\mathbb{R}_{y}^{3 N}\right), \forall s>0 .
$$

This implies the first statement of the Lemma. The asserted norm estimate (III.11) is evident from the above proof.

Lemma III.5. Let $\phi_{\beta}$ be a normalized eigenfunction of $P^{a}: P^{a} \phi_{\beta}=E_{\beta} \phi_{b}$ with eigenvalue $E_{\beta} \leq E_{\alpha}$. Then

$$
\left\langle\mathrm{I}_{a} \phi_{\alpha}, \phi_{\beta}\right\rangle_{y} \in L^{2, s}\left(\mathbb{R}_{x}^{3}\right) \quad \forall s<1 / 2,
$$

and in the case $E_{\alpha}=E_{\beta}$ we have the improved estimate

$$
\left\langle\mathrm{I}_{a} \phi_{\alpha}, \phi_{\beta}\right\rangle_{y} \in L^{2, s}\left(\mathbb{R}_{x}^{3}\right) \quad \forall s<3 / 2 .
$$

Proof: We use an explicit computation to check the case $E_{\alpha}=E_{\beta}$. In this case, Hypothesis 1 implies that

$$
\phi_{\beta}(y)=\phi_{\alpha, 1}\left(y_{1}\right) \phi_{\beta, 2}\left(y_{2}\right)
$$

where

$$
P^{a_{2}} \phi_{\beta, 2}=E_{\alpha, 2} \phi_{\beta, 2}, \quad\left\|\phi_{\beta, 2}\right\|=1
$$

Setting $\hat{x}=\frac{x}{|x|}$, we have modulo a term in $L^{2, s}\left(\mathbb{R}_{x}^{3}\right)$, for any $s<3 / 2$ and for $|x|>1$,

$$
\left\langle\mathrm{I}_{a} \phi_{\alpha}, \phi_{\beta}\right\rangle_{y}=\frac{1}{|x|^{2}}\left(\left(C_{1}+Z_{1}\right) \Delta_{2, \beta}(\hat{x})-\left(C_{2}+Z_{2}\right) \Delta_{1, \beta}(\hat{x})\right)
$$

where

$$
C_{j}=\sum_{k \in a_{j}^{\prime}} e_{k}, \quad j=1,2
$$

and

$$
\begin{aligned}
\Delta_{j, \beta}(\hat{x}) & =\sum_{k \in a_{j}^{\prime}} e_{k} \int \hat{x} \cdot y_{k} \phi_{\alpha}(y) \phi_{\beta}(y) d y \\
& =\sum_{k \in a_{j}^{\prime}} e_{k} \int \hat{x} \cdot y_{k}\left|\phi_{\alpha, 1}\left(y_{1}\right)\right|^{2} \phi_{\alpha, 2}\left(y_{2}\right) \phi_{\beta, 2}\left(y_{2}\right) d y, \quad y=\left(y_{1}, y_{2}\right)
\end{aligned}
$$


Since $C_{1}=-Z_{1}$, one has, modulo a term in $L^{2, s}\left(\mathbb{R}_{x}^{3}\right)$, for any $s<3 / 2$,

$$
\left\langle\mathrm{I}_{a} \phi_{\alpha}, \phi_{\beta}\right\rangle_{y}=-\frac{1}{|x|^{2}}\left(C_{2}+Z_{2}\right) \Delta_{1, \beta}(\hat{x})
$$

Using Hypothesis 1 (see the remark following it), we see that

$$
y^{\prime} \mapsto \sum_{k \in a_{1}^{\prime}} e_{k} \hat{x} \cdot y_{k}\left|\phi_{\alpha, 1}\left(y^{\prime}\right)\right|^{2}
$$

is an odd function of $y^{\prime}$, where $y^{\prime}=\left(y_{k} ; k \in a_{1}^{\prime}\right)$. Thus its integral vanishes and $\Delta_{1, \beta}(\hat{x})=0, \quad \forall \hat{x}$, which proves (III.14). The proof of (III.13) is similar.

We shall now localize in energy using the spectral projections for $P^{a}$. We set $2 \delta:=\operatorname{dist}\left(E_{\alpha}, \sigma\left(P^{a}\right) \backslash\right.$ $\left.\left\{E_{\alpha}\right\}\right)>0$ and denote by $\Pi_{1}$ the spectral projection of $P^{a}$ associated with $E_{\alpha}$ and by $\Pi_{2}, \Pi_{3}$ the spectral projections associated with the intervals $]-\infty, E_{\alpha}$ [and $] E_{\alpha}, \infty\left[\right.$. The projections $\Pi_{j}$ are regarded as operators in $L^{2}\left(\mathbb{R}^{3(N+1)}\right)$. It is then possible to estimate on the range of the spectral projections $\Pi_{2}, \Pi_{3}$ the resolvent $R_{a}(z)=\left(P_{a}-z\right)^{-1}$ of the Hamiltonian $P_{a}$ describing the free motion of the clusters, which was defined in (II.9). One finds

Lemma III.6. Let $\chi \in C_{0}^{\infty}(]-\delta, \delta[)$ and $u \in L^{\infty}\left(\mathbb{R}_{x}^{3}\right)$. For $j=2,3, R_{a}(\lambda) \Pi_{j} \chi\left(-\Delta_{x}-\lambda_{a}\right)$ are bounded operators and we have the weighted estimate

$$
\left\|\langle y\rangle^{s}\langle x\rangle^{s^{\prime}} R_{a}(\lambda) \Pi_{j} \chi\left(-\Delta_{x}-\lambda_{a}\right)\left(\mathrm{I}_{a} \phi_{\alpha} u\right)\right\| \leq C||\langle x\rangle^{-s^{\prime \prime}} u \|_{L^{\infty}}
$$

for all $s>0$ and for all $s^{\prime}, s^{\prime \prime}$ satisfying $s^{\prime}+s^{\prime \prime}<1 / 2$.

Proof: Setting $\psi=\mathrm{I}_{a} \phi_{a} u$, we have $\Pi_{2} \psi=\sum_{E_{\beta}<E_{\alpha}}\left\langle\psi, \phi_{\beta}\right\rangle_{L^{2}\left(\mathbb{R}_{y}^{3 N}\right)} \phi_{\beta}$, where $\left\{\phi_{\beta}\right\}$ is an orthonormal set of eigenfunctions of $P^{a}$ with eigenvalue $E_{\beta}<E_{\alpha}$. By definition of $\delta$, if $\left|\xi^{2}-\lambda_{\alpha}\right|<\delta$, then

$$
\xi^{2}+E_{\beta}-\lambda=\xi^{2}-\lambda_{\alpha}+E_{\beta}-E_{\alpha}
$$

is invertible. Thus, using the support properties of $\chi$, the function $g_{\beta}(\xi, \lambda)=\chi\left(\xi^{2}-\lambda_{\alpha}\right)\left(\xi^{2}+E_{\beta}-\lambda\right)^{-1}$ is bounded and smooth, for $E_{\beta}$ as above. Furthermore

$$
R_{a}(\lambda) \chi\left(-\Delta_{x}-\lambda_{\alpha}\right) \Pi_{2} \psi=\sum_{E_{\beta}<E_{\alpha}} g_{\beta}\left(D_{x}, \lambda\right)\left\langle\psi, \phi_{\beta}\right\rangle_{L^{2}\left(\mathbb{R}_{y}^{3 N}\right)} \phi_{\beta} .
$$

Using decay of $\phi_{\beta}$ in the variable $y$ one can apply Lemma III.5 with $s=s^{\prime}+s^{\prime \prime}<1 / 2$ to get the asserted estimate for $j=2$. For $j=3$, we have $P_{3}^{a}:=\Pi_{3} P^{a} \Pi_{3} \geq\left(E_{\alpha}+2 \delta\right) \Pi_{3}$. Applying the Fourier transformation with respect to the $\mathrm{x}$-variable, we see as above that

$$
R_{a}(\lambda) \chi\left(-\Delta_{x}-\lambda_{\alpha}\right) \Pi_{3}=\left(P_{3}^{a}-\Delta_{x}-\lambda\right)^{-1} \chi\left(-\Delta_{x}-\lambda_{\alpha}\right) \Pi_{3}
$$

is well defined as a bounded operator on $L^{2}\left(\mathbb{R}^{3(N+1)}\right)$. Applying the method of commutators, one can verify by induction that

$$
\left\|\langle y\rangle^{s}\langle x\rangle^{s^{\prime}}\left(P_{3}^{a}-\Delta_{x}-\lambda\right)^{-1} \chi\left(-\Delta_{x}-\lambda_{\alpha}\right) \Pi_{3}\langle y\rangle^{-s}\langle x\rangle^{-s^{\prime}}\right\|_{\mathcal{L}\left(L^{2}\right)} \leq C
$$

for any $s, s^{\prime} \in \mathbb{R}$. Granted this, the estimate for $j=3$ follows from the following weighted estimate on $\psi$

$$
\left\|\langle y\rangle^{s}\langle x\rangle^{s^{\prime}} \mathrm{I}_{a} \phi_{\alpha} u\right\| \leq C\|u\|_{L^{\infty}}
$$

for any $s>0, s^{\prime}<1 / 2$, which is an easy consequence of decay of $\phi_{\alpha}$ in $y$ and fall-off proportional to $|x|^{-2}$ of $\left\|\mathrm{I}_{a} \phi_{\alpha}\right\|_{y}$.

Piecing together the results of these Lemmata, we are now ready to give the 
Proof of Theorem III.3: Let $\delta>0$ be given as above and let $\lambda \in I_{\alpha} \backslash J$. We then decompose $\psi=\mathrm{I}_{a} \phi_{\alpha} u$ into 4 pieces via

$$
\psi=\sum_{j=0}^{3} \psi_{j}, \quad \psi_{0}=\left(1-\chi\left(D_{x}^{2}-\lambda_{\alpha}\right)\right) \psi, \quad \psi_{j}=\Pi_{j} \chi\left(-\Delta_{x}-\lambda_{\alpha}\right) \psi, \quad j=1,2,3 .
$$

Similarly, for $v \in L^{\infty}\left(\mathbb{R}_{x}^{3}\right)$, with $u, v$ satisfying equation (III.5), we decompose $\phi:=\mathrm{I}_{a} \phi_{\alpha} v:=\sum_{j=0}^{3} \phi_{j}$. This gives

$$
\langle R(\lambda+i \epsilon) \psi, \phi\rangle=\sum_{j, k=0}^{3}\left\langle R(\lambda+i \epsilon) \psi_{j}, \phi_{k}\right\rangle
$$

For $j=0,1$, we get from Lemma III.4 and III.5 that $\psi_{j}, \phi_{j} \in L^{2, s}\left(\mathbb{R}^{3(N+1)}\right), \quad \forall s<3 / 2$. This gives for $j, k=0,1$, using the weighted estimate for the resolvent,

$$
\begin{aligned}
\left|\left\langle R(\lambda+i \epsilon) \psi_{j}, \phi_{k}\right\rangle\right| & \leq C\left\|\langle(x, y)\rangle^{s} \psi_{j}\right\| \|\left\langle\langle(x, y)\rangle^{s} \phi_{k} \|\right. \\
& \leq C_{1}\left\|\langle x\rangle^{-s^{\prime}} u\right\|_{L^{\infty}}\left\|\langle x\rangle^{-s^{\prime}} v\right\|_{L^{\infty}}
\end{aligned}
$$

for any $s>1 / 2,0<s^{\prime}<3 / 2-s$. This estimate and those below are all uniform in $\epsilon \in[-1,0[\cup] 0,1]$. In the case $j=0,1$, but $k=2,3$, we decompose further using the resolvent equation

$$
R(\lambda+i \epsilon)=R_{a}(\lambda+i \epsilon)-R_{a}(\lambda+i \epsilon) \mathrm{I}_{a} R(\lambda+i \epsilon) .
$$

This gives

$$
\begin{aligned}
\left|\left\langle R(\lambda+i \epsilon) \psi_{j}, \phi_{k}\right\rangle\right| & \leq C\left(\left\|\langle x\rangle^{s} \psi_{j}\right\|\left\|\mid\langle x\rangle^{-s} R_{a}(\lambda-i \epsilon) \phi_{k}\right\|+\left\|\langle(x, y)\rangle^{s} \psi_{j}\right\|\left\|\langle x\rangle^{-1+s} R_{a}(\lambda-i \epsilon) \phi_{k}\right\|\right) \\
& \leq C_{1}\left\|\langle x\rangle^{-s^{\prime}} u\right\|_{L^{\infty}}\left\|\langle x\rangle^{-s^{\prime}} v\right\|_{L^{\infty}},
\end{aligned}
$$

for any $s>1 / 2,0<s^{\prime}<3 / 2-s$. Here we have used the weighted estimate on the resolvent $R(\lambda \pm i \epsilon)$ and on $\psi_{j}$, for $j=0,1$, - as explained after equation (III.19) - to estimate the contribution of $\psi_{j}$ and we have used Lemma III. 6 to estimate the contribution of $\phi_{k}$. Interchanging $j, k$ we obtain the same estimates for the other cross terms $j=2,3$ and $k=0,1$.

Finally, to treat the case $j, k=2,3$, we iterate the resolvent equation once more:

$$
R(\lambda+i \epsilon)=R_{a}(\lambda+i \epsilon)-R_{a}(\lambda+i \epsilon) \mathrm{I}_{a} R_{a}(\lambda+i \epsilon)+R_{a}(\lambda+i \epsilon) \mathrm{I}_{a} R(\lambda+i \epsilon) \mathrm{I}_{a} R_{a}(\lambda+i \epsilon) .
$$

The first 2 terms on the rhs of this equation are easily handled by Lemma III.6 and give

$$
\begin{aligned}
\left|\left\langle R_{a}(\lambda+i \epsilon) \psi_{j}, \phi_{k}\right\rangle\right| & \leq C\left\|\langle x\rangle^{-s^{\prime}} u\right\|_{L^{\infty}}\left\|\langle x\rangle^{-s^{\prime}} v\right\|_{L^{\infty}}, & & \forall s^{\prime}<1 / 2 \\
\left|\left\langle R_{a}(\lambda+i \epsilon) \mathrm{I}_{a} R_{a}(\lambda+i \epsilon) \psi_{j}, \phi_{k}\right\rangle\right| & \leq C\left\|\langle x\rangle^{-s^{\prime}} u\right\|_{L^{\infty}}\left\|\langle x\rangle^{-s^{\prime}} v\right\|_{L^{\infty}}, & & \forall s^{\prime}<1 .
\end{aligned}
$$

For the third term on the rhs of equation III.22 we obtain, again via Lemma III.6,

$$
\begin{aligned}
\left|\left\langle R_{a}(\lambda+i \epsilon) \mathrm{I}_{a} R(\lambda+i \epsilon) \mathrm{I}_{a} R_{a}(\lambda+i \epsilon) \psi_{j}, \phi_{k}\right\rangle\right| & \leq C\left\|\langle x\rangle^{-1+s} R_{a}(\lambda+i \epsilon) \psi_{j}\right\|\left\|\mid\langle x\rangle^{-1+s} R_{a}(\lambda-i \epsilon) \phi_{k}\right\| \\
& \leq C\left\|\langle x\rangle^{-s^{\prime}} u\right\|_{L^{\infty}}\left\|\langle x\rangle^{-s^{\prime}} v\right\|_{L^{\infty}},
\end{aligned}
$$

for any $s>1 / 2,0<s^{\prime}<3 / 2-s$. Choosing $s$ arbitrarily close to $1 / 2$ and adding equations (III.20), (III.21), (III.23) and (III.24) proves the uniform boundedness in Theorem III.3. To see the existence of the weak limit, we use the same decompositions. The desired result follows from Lemma III.5, Lemma III.6 and the existence of the boudary values $R(\lambda \pm i 0)$ as operator from $L^{2, s}$ to $L^{2,-s}$ for $s>1 / 2$. 


\section{Born-Oppenheimer approximation of $\sigma_{\alpha}$}

This section is devoted to a sketch of the proof of Theorem II.3. Within this section, we shall always assume Hypotheses 1,2 and 3 and we reinsert the parameter $h$ which is defined in (II.2).

To study $\sigma_{\alpha}$ given by (II.26), we need various approximations and estimates for the boundary value of the resolvent and for the function $\mathrm{I}_{a} e_{\alpha}$, which are collected in the following

Proposition IV.1. Let $\Gamma$ be the $h$-dependent set of all possible collisions (defined in A.5) and let $\chi$ be an $h$-dependent smooth function on $\mathbb{R}^{3(N+1)}$, equal to one on some conic neighborhood of $\Gamma$, and equal to zero on some bigger conic neighborhood. For any $s \geq 0$, we have, uniformly w.r.t. $h$,

$$
\begin{aligned}
\chi \mathrm{I}_{a} \phi_{\alpha} & \in L_{s}^{2}\left(\mathbb{R}^{3(N+1)}\right), \\
\left\|(1-\chi) \mathrm{I}_{a} \phi_{\alpha}\right\|_{y} & =\mathcal{O}\left(\langle x\rangle^{-2}\right),
\end{aligned}
$$

where $\|\cdot\|_{y}$ denotes the norm on $L^{2}\left(\mathbb{R}_{y}^{3 N}\right)$. For $|x|>1$, uniformly w.r.t. $h$,

$$
\begin{aligned}
\left\|\Pi(x ; h) \mathrm{I}_{a}(x, \cdot ; h) \phi_{\alpha}\right\|_{y} & =\mathcal{O}\left(|x|^{-4}\right), \\
\mathrm{I}_{\mathrm{eff}}(x):=\lambda_{1}(x ; 0)-E_{\alpha}(0) & =\mathcal{O}\left(|x|^{-4}\right), \\
\left\|\Pi(x ; h)\left(\mathrm{I}_{a}(x, ; h)-\mathrm{I}_{\mathrm{eff}}(x)\right) \phi_{\alpha}\right\|_{y} & =\mathcal{O}\left(h^{2}|x|^{-4}\right)+\mathcal{O}\left(|x|^{-5}\right), \\
\left\|\Pi(x ; h)\left(\mathrm{I}_{a}(x, ; h)-\hat{\mathrm{I}}_{\mathrm{eff}}(x)\right) \phi_{\alpha}\right\|_{y} & =\mathcal{O}\left(h^{2}|x|^{-4}\right)+\mathcal{O}\left(|x|^{-5}\right),
\end{aligned}
$$

Furthermore, the smooth function $\mathbb{R}^{3} \backslash\{0\} \ni x \mapsto \Pi(x ; h)$ has the following properties. There exists $\mu>0$ such that, uniformly w.r.t. $x$ and $h$,

$$
\sum_{|\beta| \leq 2}\langle x\rangle^{4+|\beta|}\left\|e^{\mu\langle y\rangle} \Pi(x ; h) \partial_{x}^{\beta}\left(\Pi(x ; h)-\Pi_{0}(h)\right) \Pi_{0}(h)\right\|_{\mathcal{L}\left(L^{2}\left(\mathbb{R}_{y}^{3 N}\right)\right)}=\mathcal{O}(1) .
$$

Note that (IV.7) remains true if the first projector $\Pi(x ; h)$ is replaced by $\Pi_{0}(h)$. The resolvents satisfy, for all $s>1 / 2$ and locally uniformly for $\lambda \in J$,

$$
\begin{aligned}
\left\|\langle x-l(y)\rangle^{-s} \mathrm{R}(\lambda \pm i 0)\langle x-l(y)\rangle^{-s}\right\|+\left\|\langle x\rangle^{-s} \mathrm{R}^{\mathrm{AD}}(\lambda \pm i 0 ; h)\langle x\rangle^{-s}\right\| & =\mathcal{O}\left(h^{-1}\right) \\
\left\|\langle x-l(y)\rangle^{-s} \mathrm{R}(\lambda \pm i 0 ; h) \hat{\Pi}\right\| & =\mathcal{O}(1) \\
\left\|\langle x\rangle^{-s} \Pi\left(\mathrm{R}(\lambda \pm i 0)-\mathrm{R}^{\mathrm{AD}}(\lambda \pm i 0 ; h)\right) \Pi\langle x\rangle^{-s}\right\| & =\mathcal{O}(1)
\end{aligned}
$$

uniformly in $\left.h \in] 0, h_{0}\right]$.

Proof: (IV.1) follows from the exponential decay of the eigenfunctions $\phi_{\alpha}(h)$, which is uniform w.r.t. $h$. According to Appendix A, equation (IV.2) holds for $|x|>1$ and uniformly w.r.t. $h$, and

$$
\left\|\left(\Pi_{0}(h)+\Pi_{0}(0)\right)\left(\mathrm{I}_{a}(x ; h)+\mathrm{I}_{a}(x ; 0)\right)\left(\Pi_{0}(h)+\Pi_{0}(0)\right)\right\|_{y}=\frac{A(\hat{x} ; h)}{|x|^{5}}+\mathcal{O}\left(|x|^{-6}\right),
$$

where $\hat{x}=x /|x|$ and $A(\hat{x} ; h)$ is uniformly bounded as $h \rightarrow 0$. Furthermore, the operator $\langle x\rangle^{2} \mathrm{I}_{a}(x ; h) \Pi_{0}(h)$ is uniformly bounded. Using this fact, we can show, as in [KMW2], that

$$
\sum_{|\beta| \leq 2}\langle x\rangle^{2+|\beta|}\left\|e^{\mu\langle y\rangle} \partial_{x}^{\beta}\left(\Pi(x ; h)-\Pi_{0}(h)\right)\right\|_{y}=\mathcal{O}(1) .
$$

Using (IV.12) and (IV.11), we obtain

$$
\begin{aligned}
\Pi(x ; h) \mathrm{I}_{a}(x ; h) \Pi_{0}(h) & =\left(\Pi(x ; h)-\Pi_{0}(h)\right) \mathrm{I}_{a}(x ; h) \Pi_{0}(h)+\Pi_{0}(h) \mathrm{I}_{a}(x ; h) \Pi_{0}(h) \\
& =\mathcal{O}\left(|x|^{-4}\right) .
\end{aligned}
$$

Next, we show that

$$
\lambda_{1}(x ; h)-E_{\alpha}(h)=\hat{\mathrm{I}}_{\mathrm{eff}}(x)+\mathcal{O}\left(h^{2}|x|^{-4}\right)+\mathcal{O}\left(|x|^{-5}\right)
$$


Using (IV.12), we first note that, since the eigenfunction $\phi_{\alpha}(h)$ is normalized,

$$
\left\|\Pi(x ; h) \phi_{\alpha}(h)\right\|_{y}^{2}=1+\mathcal{O}\left(|x|^{-2}\right) .
$$

Thus, according to (IV.11), for $|x|$ large enough and writing $\langle\cdot, \cdot\rangle_{y}$ for the scalar product in $L^{2}\left(\mathbb{R}_{y}^{3 N}\right)$, we have

$$
\begin{aligned}
\lambda_{1}(x ; h)-E_{\alpha}(h) & =\left\langle\Pi(x ; h) \phi_{\alpha}(h), \mathrm{I}_{a}(x ; h) \Pi(x ; h) \phi_{\alpha}(h)\right\rangle_{y} /\left\|\Pi(x ; h) \phi_{\alpha}(h)\right\|_{y}^{2} \\
& =\left\langle\Pi(x ; h) \phi_{\alpha}(h), \mathrm{I}_{a}(x ; h) \Pi(x ; h) \phi_{\alpha}(h)\right\rangle_{y}+\mathcal{O}\left(|x|^{-6}\right) \\
& =2 \Re\left\langle\left(\Pi(x ; h)-\Pi_{0}(h)\right) \phi_{\alpha}(h), \mathrm{I}_{a}(x ; h) \Pi(x ; h) \phi_{\alpha}(h)\right\rangle_{y}+\mathcal{O}\left(|x|^{-5}\right) \\
& =2 \Re\left\langle\left(\Pi(x ; h)-\Pi_{0}(h)\right) \phi_{\alpha}(h), \mathrm{I}_{a}(x ; h) \phi_{\alpha}(h)\right\rangle_{y}+\mathcal{O}\left(|x|^{-5}\right)
\end{aligned}
$$

Next, we use the following lemma, which will be proved after the present proof.

Lemma IV.2. Setting $\hat{R}_{a}(z, h)=\left(P^{a}(h) \hat{\Pi}_{0}(h)-z\right)^{-1} \hat{\Pi}_{0}(h)$ and $\hat{R}_{a}(h)=\hat{R}_{a}\left(E_{\alpha}(h), h\right)$ (as in equation (II.34)), we have, for $|x|$ large enough and uniformly w.r.t. $h$,

$$
\begin{aligned}
2 \Re\left\langle\left(\Pi(x ; h)-\Pi_{0}(h)\right) \phi_{\alpha}(h), \mathrm{I}_{a}(x ; h) \phi_{\alpha}(h)\right\rangle_{y} \\
\quad=-2\left\langle\hat{R}_{a}(h) \hat{\Pi}_{0}(h) C(\hat{x}, y) \phi_{\alpha}(h), \hat{\Pi}_{0}(h) C(\hat{x}, y) \phi_{\alpha}(h)\right\rangle_{y} \cdot|x|^{-4}+\mathcal{O}\left(|x|^{-5}\right) .
\end{aligned}
$$

In particular, the two forms of the effective potential satisfy equation (II.36), i.e.

$$
\left|\mathrm{I}_{\text {eff }}(x)-\hat{\mathrm{I}}_{\text {eff }}(x)\right|=\mathcal{O}\left(|x|^{-5}\right), \quad \text { as }|x| \rightarrow \infty .
$$

Furthermore, the first term on the rhs of (IV.16) is negative, for all $x \neq 0$, if the cluster $a_{2}$ is not neutral.

Using Lemma IV.2, we obtain (IV.14). By a Taylor expansion w.r.t. $h$ and using the previous estimates,

$$
\begin{aligned}
\Pi(x ; h) \mathrm{I}_{a}(x ; h) \Pi_{0}(h) & =\Pi(x ; h) \mathrm{I}_{a}(x ; 0) \Pi_{0}(h)+\mathcal{O}\left(h|x|^{-6}\right) \\
& =\Pi(x ; 0) \mathrm{I}_{a}(x ; 0) \Pi_{0}(0)+\mathcal{O}\left(h^{2}|x|^{-5}\right)+\mathcal{O}\left(|x|^{-6}\right) \\
& =\Pi(x ; 0)\left(\lambda(x ; 0)-E_{\alpha}(0)\right) \Pi_{0}(0)+\mathcal{O}\left(h^{2}|x|^{-5}\right)+\mathcal{O}\left(|x|^{-6}\right) \\
& =\Pi(x ; h)\left(\lambda(x ; 0)-E_{\alpha}(0)\right) \Pi_{0}(h)+\mathcal{O}\left(h^{2}|x|^{-5}\right)+\mathcal{O}\left(|x|^{-6}\right) .
\end{aligned}
$$

We then have proved (IV.5). Using (IV.15), we derive (IV.6) from (IV.5).

Finally, we follow the arguments in [KMW2] to derive (IV.7) from (IV.13). Still following [KMW2], we obtain resolvent estimates with the weight $\langle x-l(y)\rangle$. As already remarked in [KMW2], $\langle x\rangle^{-s} \Pi(x)\langle x-l(y)\rangle^{s}$ is uniformly bounded. Thus we may replace this weight by $\langle x\rangle$ if $\Pi$ is present. We do this for the second term in (IV.8) and in (IV.10).

Proof of Lemma IV.2: Equation(II.36) simply follows from (IV.15) and(IV.16), for $h=0$. To prove (IV.16), we write the projections as contour integrals. Let $\Gamma$ a complex contour enclosing $E_{\alpha}(h)$ and $\lambda_{1}(x ; h)$ for $h$ sufficiently small and $|x|$ sufficiently large. For brevity, we shall now notationally suppress the dependence on $h$. We rewrite the lhs of equation (IV.16) as

$$
\begin{aligned}
\operatorname{lhs}(\mathrm{IV} .16) & =2 \Re\left\langle\left(\left(\mathrm{P}_{e}(x)-\bar{z}\right)-\left(\mathrm{P}^{a}-\bar{z}\right)\right) \phi_{\alpha}, \frac{1}{2 i \pi} \oint_{\Gamma}\left(\left(\mathrm{P}_{e}(x)-z\right)^{-1}-\left(\mathrm{P}^{a}(x)-z\right)^{-1}\right) d z \phi_{\alpha}\right\rangle_{y} \\
& =-2 \Re \frac{1}{2 i \pi} \oint_{\Gamma} d z\left(\left(E_{\alpha}-z\right)\left\langle\left(\mathrm{P}_{e}(x)-z\right)^{-1} \phi_{\alpha}, \phi_{\alpha}\right\rangle_{y}+\left(E_{\alpha}-z\right)^{-1}\left\langle\mathrm{I}_{a}(x) \phi_{\alpha}, \phi_{\alpha}\right\rangle_{y}\right) \\
& =-2 \Re \frac{1}{2 i \pi} \oint_{\Gamma} d z\left(E_{\alpha}-z\right)\left\langle\left(\mathrm{P}_{e}(x)-z\right)^{-1} \phi_{\alpha}, \phi_{\alpha}\right\rangle_{y}+\mathcal{O}\left(|x|^{-5}\right),
\end{aligned}
$$

by (IV.11). So we need to compute $\Pi_{0} R_{e}(z) \Pi_{0}$, where $R_{e}(z)=\left(\mathrm{P}_{e}(x)-z\right)^{-1}$. To this end, we use the resolvent equation

$$
R_{e}(z)=R_{a}(z)-R_{a}(z) I_{a} R_{a}(z)+R_{a}(z) I_{a}(z) R_{e}(z) I_{a} R_{a}(z)
$$


which gives

$$
\Pi_{0} R_{e}(z) \Pi_{0}=R_{a}(z) \Pi_{0}+R_{a}(z) \Pi_{0} I_{a} \hat{\Pi}_{0} R_{e}(z) \hat{\Pi}_{0} I_{a} \Pi_{0} R_{a}(z)+\mathcal{O}\left(|x|^{-5}\right)
$$

Inserting these estimates into (IV.17) and using Appendix C and (IV.11) again, we arrive at (IV.16) with $\hat{R}_{a}(h)$ replaced by $\hat{\Pi}_{0} R_{e}\left(E_{\alpha}(h)\right) \hat{\Pi}_{0}$. But

$$
\left\|\hat{\Pi}_{0}\left(R_{e}\left(E_{\alpha}\right)-R_{a}\left(E_{\alpha}\right)\right) \hat{\Pi}_{0} C(\hat{x} ; y) \phi_{\alpha}\right\|_{y}=\mathcal{O}\left(|x|^{-2}\right),
$$

uniformly w.r.t. h. This follows from a Neumann expansion of $R_{e}(z)$, exponential decay of $\phi_{\alpha}$, uniform boundedness of the weighted reduced resolvent $\langle y\rangle^{M} \hat{R}_{a}\langle y\rangle^{-M}$, for any $M \geq 0$, combined with $\left\|I_{a}\langle y\rangle^{-M}\right\|_{y}=$ $\mathcal{O}\left(|x|^{-2}\right)$. This proves equation (IV.20) and thus (IV.16).

Since $\hat{\mathbf{R}}_{a}\left(E_{\alpha}(h)\right) \geq b>0$, uniformly w.r.t. $h$, the first term on the rhs of (IV.16) is bounded above by $-b\left\|\hat{\Pi}_{0} C(\hat{x} ; y) \phi_{\alpha}(h)\right\|^{2} /|x|^{4}$. Since

$$
\left\|\Pi_{0} C(\hat{x} ; \cdot) \phi_{\alpha}(h)\right\|_{y}^{2}=0
$$

by the rotational invariance of $\phi_{\alpha, 1}$ (see Appendix A), we have

$$
\left\|\hat{\Pi}_{0} C(\hat{x} ; y) \phi_{\alpha}(h)\right\|_{y}^{2}=\left\|C(\hat{x} ; y) \phi_{\alpha}(h)\right\|_{y}^{2}>0 .
$$

Recall from (II.26) that

$$
\sigma_{\alpha}(\lambda, \omega ; h)=\frac{h^{-1}}{n_{\alpha}(\lambda ; h)} \operatorname{Im}\left\langle\mathrm{R}(\lambda+i 0 ; h) \mathrm{I}_{a} e_{\alpha}, \mathrm{I}_{a} e_{\alpha}\right\rangle .
$$

In view of the adiabatic approximation of the resolvent, we introduce

$$
\sigma_{\mathrm{ad}}(\lambda, \omega ; h):=\frac{h^{-1}}{n_{\alpha}(\lambda ; h)} \operatorname{Im}\left\langle\Pi^{\mathrm{AD}}(\lambda+i 0 ; h) \Pi I_{a} e_{\alpha}, I_{a} e_{\alpha}\right\rangle .
$$

$\sigma_{\text {ad }}$ is almost the total cross-section for the scattering process of the pair of operators $\left(P^{A D}(h), \Pi(h)\left(-h^{2} \Delta_{x}+\right.\right.$ $\left.\left.E_{\alpha}(h)\right) \Pi(h)\right)$, as shown in [Jec]. It thus should be a good approximation for $\sigma_{\alpha}$. Indeed, we claim that

Proposition IV.3. For all $\epsilon>0$ small enough, there exists some $C_{>} 0$ such that, for all $h>0$ sufficiently small and locally uniformly in $(\lambda, \omega) \in J \times \mathbb{S}^{2}$,

$$
\sigma_{\alpha}(\lambda, \omega ; h)=\sigma_{\text {ad }}(\lambda, \omega ; h)+\mathcal{O}\left(h^{-2 / 3+(1 / 2-\epsilon)}\right) .
$$

With the estimates given in Proposition IV.1, the proof of Proposition IV.3 follows the same arguments as in [Jec].

The adiabatic operator $P^{A D}(h)$ is equal to $\Pi(h)\left(-h^{2} \Delta_{x}+\lambda_{1}(x, h)\right) \Pi(h)$ in $\mathcal{O}_{\lambda_{0}}$ which looks like a twobody Schrödinger operator with operator-valued potential. We can use the methods of [RT] and [Jec] to prove that

$$
\sigma_{\mathrm{ad}}(\lambda, \omega ; h)=\mathcal{O}\left(h^{-2 / 3}\right)
$$

and that

$$
\sigma_{\mathrm{ad}}(\lambda, \omega ; h)=4 \int_{H_{\omega}} \sin ^{2}\left(\frac{1}{4 n_{\alpha}(\lambda ; 0) h} \int_{-\infty}^{+\infty} \mathrm{I}_{\mathrm{eff}}\left(x_{\omega}+u \omega\right) d u\right) d x_{\omega}+\mathcal{O}\left(h^{-2 / 3+\epsilon_{0}}\right),
$$

which, according to Proposition IV.3, gives (II.39) for I $=\mathrm{I}_{\text {eff. }}$. Since the potential $\hat{\mathrm{I}}_{\text {eff }}$ has the same properties as $\mathrm{I}_{\text {eff }}$ and $\hat{\mathrm{I}}_{\text {eff }}-\mathrm{I}_{\text {eff }}=\mathcal{O}\left(|x|^{-5}\right.$ ) (see Proposition IV.1), we can show that (IV.24) still holds with $\mathrm{I}_{\text {eff }}$ replaced by $\hat{\mathrm{I}}_{\text {eff }}$. We thus obtain the formula (IV.24) with $\hat{\mathrm{I}}_{\text {eff }}$, which is (II.39) for I $=\hat{\mathrm{I}}_{\text {eff }}$.

Now we assume that $C_{2}+Z_{2} \neq 0$. To show that $\sigma_{\text {ad }}$ (and thus $\sigma_{\alpha}$ ) is exactly of order $h^{-2 / 3}$, we estimate as in [Jec] the integral in (II.39) for I $=\hat{\mathrm{I}}_{\text {eff }}$. Recall that $\hat{\mathrm{I}}_{\text {eff }}(x)$ is of the form $A(\hat{x} ; 0)|x|^{-4}$ (see (II.37)) and 
that $A(\hat{x} ; 0)$ is everywhere negative by Lemma IV.2 since $C_{2}+Z_{2} \neq 0$. Thanks to this form, one can show as in $[\mathrm{Y}]$ that, for some $h$-independent constant $b \neq 0$,

$$
4 \int_{H_{\omega}} \sin ^{2}\left(\frac{1}{4 n_{\alpha}(\lambda ; 0) h} \int_{-\infty}^{+\infty} \hat{\mathrm{I}}_{\mathrm{eff}}\left(x_{\omega}+u \omega\right) d u\right) d x_{\omega}=b h^{-2 / 3} \int_{\mathbb{S}_{\omega}^{1}}|\Omega(\omega, \tau)|^{2 / 3} d \tau,
$$

where $\mathbb{S}_{\omega}^{1}$ is the unit sphere in $H_{\omega}$ and where $\Omega$ is given by

$$
\Omega(\omega, \tau)=\int_{0}^{\pi} \hat{\mathrm{I}}_{\mathrm{eff}}(\cos \theta \omega+\sin \theta \tau) \sin ^{2} \theta d \theta
$$

for $\tau \in \mathbb{S}_{\omega}^{1}$. By Lemma IV.2 we know that the integrand - and thus $\Omega$ - is negative everywhere. Thus the rhs of (IV.25) is exactly of order $h^{-2 / 3}$.

\section{A Expansion of the potentials}

In this section we collect the relevant expansions of the Coulomb interactions for atom-ion scattering, which involve the effective dipole moments and quadrupole moments of the two clusters $a_{1}, a_{2}$. They are certainly well known in the physics literature. For the sake of the reader, we state them as

Lemma A.1. Let $\alpha=\left(a, E_{\alpha}(h), \phi_{\alpha}(h)\right)$ be a scattering channel satisfying Hypothesis 1. Then

$$
\begin{aligned}
\left\|\mathrm{I}_{a}(x, h) \phi_{\alpha}(h)\right\|_{y} & =\mathcal{O}\left(\langle x\rangle^{-2}\right), \\
\left\|\left(\mathrm{I}_{a}-\frac{C(\hat{x}, \cdot)}{|x|^{2}}\right) \phi_{\alpha}(h)\right\|_{y} & =\mathcal{O}\left(\langle x\rangle^{-3}\right), \quad C(\hat{x}, y)=\left(C_{2}+Z_{2}\right) \sum_{l \in a_{1}^{\prime}} e_{l} \hat{x} \cdot y_{l}, \\
\left\langle\phi_{\alpha}(h), \mathrm{I}_{\mathrm{a}}(x ; h) \phi_{\alpha}(h)\right\rangle_{y} & =\mathcal{O}\left(\langle x\rangle^{-3}\right),
\end{aligned}
$$

uniformly w.r.t. $h$, for $0 \leq h \leq h_{0}$. Assuming in addition that $\alpha$ satisfies Hypothesis 2, we even have the stronger estimate

$$
\left\langle\phi_{\alpha}(h), \mathrm{I}_{\mathrm{a}}(x ; h) \phi_{\alpha}(h)\right\rangle_{y}=\mathcal{O}\left(\langle x\rangle^{-5}\right),
$$

uniformly w.r.t. $h$, for $0 \leq h \leq h_{0}$.

Proof: Because of the Coulomb singularities, we separate the contribution of collisions. Let $\Gamma$ be the (h-dependent ) set of all possible collisions, that is

$$
\Gamma:=\left\{(x, y) \in \mathbb{R}^{3(N+1)} ; \exists l \in a_{1}^{\prime}, \exists j \in a_{2}^{\prime}, \begin{array}{rll}
x=-y_{l}+l(y) & \text { or } & x=y_{j}-y_{l}+l(y) \\
\text { or } \quad x=l(y) & \text { or } & x=y_{j}+l(y)
\end{array}\right\} .
$$

Let $\chi \in C^{\infty}\left(\mathbb{R}^{3(N+1)}\right)$ such that $0 \leq \chi \leq 1, \chi$ equals 1 on a small conic neighborhood of $\Gamma$, and $\chi$ equals 0 outside a slightly bigger conic neighborhood. We also demand that $\chi$ is even in $y$. Thanks to the exponential decay (uniformly w.r.t. $h$ ) of the eigenfunctions $\phi_{\alpha}(h)$, we have

$$
\left\|\chi(x, y ; h)\langle y\rangle^{L} \mathrm{I}_{\mathrm{a}}(x ; h) \phi_{\alpha}(h)\right\|_{y}=\mathcal{O}\left(\langle x\rangle^{-M}\right), \quad \forall L, M \in \mathbb{N} .
$$

Thus, we only have to estimate the contribution of the regular part

$$
\mathrm{I}_{\mathrm{reg}}(\phi):=\left\langle\phi_{\alpha}(h), \tilde{\mathrm{I}}_{\mathrm{a}}(x ; h) \phi_{\alpha}(h)\right\rangle_{y}, \quad \tilde{\mathrm{I}}_{\mathrm{a}}(x ; h):=(1-\chi(x, y ; h)) \mathrm{I}_{\mathrm{a}}(x ; h) .
$$

According to (II.8), we want to expand terms of the form

$$
|x+\tilde{l}(y)|^{-1}=|x|^{-1} \cdot|\hat{x}+\tilde{l}(y) /| x||^{-1}
$$


for large $|x|$. To this end, we use a Taylor expansion at zero of the function $f: \mathbb{R} \ni r \mapsto|u+r v|^{-1}$ for non-zero vectors $u, v \in \mathbb{R}^{3}$. More precisely, one obtains by Taylor expansion, that for each $r \in \mathbb{R}$, there exists some $\theta \in] 0 ; 1[$ such that

$$
f(r)=\sum_{k=0}^{3} \frac{r^{k}}{k !} f^{(k)}(0)+\frac{r^{4}}{4 !} f^{(k)}(\theta r)
$$

We observe that the first order term (the term in $|x|^{-1}$ ) of the expansion of $\mathrm{I}_{\mathrm{reg}}(\phi)$ vanishes by neutrality of $a_{1}$ (cf. (II.21)). The second order term is given by

$$
-\left\langle\phi_{\alpha}(h), C(\hat{x}, \cdot) \phi_{\alpha}(h)\right\rangle_{y} \cdot|x|^{-2}, \quad C(\hat{x}, y)=\left(C_{2}+Z_{2}\right) \sum_{l \in a_{1}^{\prime}} e_{l} \hat{x} \cdot y_{l}
$$

where $C_{2}=\sum_{l \in a_{2}^{\prime}} e_{l}$ is the electronic charge of $a_{2}$ and where we have used an estimate similar to(A.6) to get

$$
\left\|\chi(x, y ; h) \phi_{\alpha}(h) C(\hat{x}, \cdot) \phi_{\alpha}(h)\right\|_{y}=\mathcal{O}\left(\langle x\rangle^{-M}\right), \forall M \in \mathbb{N} .
$$

By the reflexion symmetry, we see that the second order term also vanishes. The rest of the expansion is seen to be $O\left(|x|^{-3}\right)$, uniformly w.r.t. $h$. In view of equation (A.6), this proves (A.2) and (A.3). The proof of (A.1) is similar and involves a non-vanishing term of second order. Next we shall prove the estimate (A.4). It crucially depends on the full rotational symmetry of the wave function $\phi_{\alpha}=\phi_{\alpha, 1} \phi_{\alpha, 2}$ in both clusters (which is a consequence of Hypothesis 2). For convenience, we choose the cut-off $\chi$ in such a way that the new cut-off also has the same symmetry properties. To this end, we consider $\tilde{\Gamma}$, defined as the union of the orbits under the action $o: y \rightarrow o \cdot y=\left(o \cdot y_{1}, \cdots, o \cdot y_{N}\right)$ of $O(3, \mathbb{R})$ on the $y$-variables of each point in $\Gamma$ (for $h=0$ ). As before, we construct a cut-off $\tilde{\chi} \in C^{\infty}\left(\mathbb{R}^{3(N+1)}\right)$ such that $0 \leq \tilde{\chi} \leq 1, \tilde{\chi}$ equals 1 on a small conic neighborhood of $\tilde{\Gamma}$, and $\tilde{\chi}$ equals 0 outside a slightly bigger conic neighborhood. Notice that, on the support of $\tilde{\chi}$, the previous properties are preserved since $|x|$ and $|y|$ are equivalent there. Thus (A.6) holds in this case also, and it again suffices to estimate the regular part defined in (A.7). Obviously, the first and second order term of the expansion are zero. Expanding further, we find that the third order term is

$$
\left\langle\phi_{\alpha}(h), F_{3}(\hat{x}, y) \phi_{\alpha}(h)\right\rangle \cdot|x|^{-3}
$$

where we have estimated the contribution of the region cut out by $\tilde{\chi}$ as above and where

$$
F_{3}(\hat{x}, y)=-\left(C_{2}+Z_{2}\right) \sum_{l \in a_{1}^{\prime}}\left(e_{l}\left|y_{l}\right|^{2}-3\left(y_{l} \cdot \hat{x}\right)^{2}\right)+2 \sum_{l \in a_{1}^{\prime}, j \in a_{2}^{\prime}} e_{l} e_{j}\left(y_{l} \cdot y_{j}-3\left(y_{l} \cdot \hat{x}\right)\left(y_{j} \cdot \hat{x}\right)\right) .
$$

By the rotation symmetry of Coulomb potentials, we can replace in (A.10) $\hat{x}$ by the canonical basis vectors $b_{1}, b_{2}, b_{3}$ of $\mathbb{R}^{3}$. Since $\sum_{k} F_{3}\left(b_{k}, y\right)=0$, it follows that the third order term also vanishes. For the fourth order term, we get

$$
\left\langle\phi_{\alpha}(h), F_{4}(\hat{x}, y) \phi_{\alpha}(h)\right\rangle \cdot|x|^{-4}
$$

where the function $F_{4}$ satisfies

$$
F_{4}\left(\hat{x}, y_{1},-y_{2}\right)=-F_{4}\left(\hat{x}, y_{1}, y_{2}\right), \quad y=\left(y_{1}, y_{2}\right)
$$

since it is homogeneous of degree 3 in $y$. By Hypothesis 2 the eigenvalue $E_{\alpha, 2}$ is simple and $\phi_{\alpha, 2}$ is invariant under the reflection $y_{2} \mapsto-y_{2}$. Thus the fourth order term is zero, and a standard application of Taylor's theorem (A.8) shows that the remainder of the expansion is $O\left(|x|^{-5}\right)$, uniformly w.r.t. $h$.

Acknowledgements. X.P. Wang thanks the organizors of the Bologna APTEX International Conference, September 1999, for their invitation and hospitality. 


\section{References}

[A] S.Agmon: Lectures on Exponential Decay of Solutions of Second-Order Elliptic Equations. Princeton University Press, 1982.

[CT] J.M.Combes, A.Tip: Properties of the scattering amplitude for electron-atom collisions. Ann. I.H.P., vol. $40, n^{\circ} 2,1984$, p. 117-139.

[DG] J.Derezinsky, C.Gerard: Scattering Theory of Classical and Quantum N-Particle Systems. Springer, (1997).

[ES] V.Enss, B.Simon: Finite Total Cross-Section in Nonrelativistic Quantum Mechanics. Commun. Math. Phys. 76, 177-209 (1980).

[HV] W.Hunziker, E.Vock: Stability of Schrödinger Eigenvalue Problems. Comm. Math. Phys. 83, 281-302, 1982.

[I1] H.T.Ito: High-energy behavior of the total scattering cross sections for 3-body quantum systems. Publ. Res. Inst. Math. Sci. 29, No. 5, 803-832 (1993).

[I2] H.T.Ito: The semiclassical asymptotics of the total cross-sections for elastic scattering for $N$-body systems. J.Math. Kyoto Univ. 33, No. 4, 1143-1164 (1993).

[Jec] Th.Jecko: Approximation de Born-Oppenheimer de sections efficaces totales diatomiques. To appear in Asymptotic Analysis.

[KMW1] M.Klein, A.Martinez, X.P.Wang: On the Born-Oppenheimer Approximation of Wave Operators in Molecular Scattering Theory. Commun. Math. Phys. 152, 73-95, 1993.

[KMW2] M.Klein, A.Martinez, X.P.Wang: On the Born-Oppenheimer Approximation of Wave Operators II: Singular Potentials. J. Math. Phys. Vol. 38, No 3, 1373-1396, 1997.

[PSS] P.Perry, B.Simon, I.Sigal: Spectral analysis of N-body Schrödinger operators. Ann. of Math. 114, 519-567, 1981.

[Ra] A.Raphaelian: Ion-Atom Scattering within the Born-Oppenheimer framework. Dissertation TU Berlin, 1986.

[RT] D.Robert, H.Tamura: Semiclassical estimates for resolvents and asymptotics for total cross-section. Ann. IHP 46, 415-442, 1987.

[RW] D.Robert, X.P.Wang: Pointwise Semiclassical Asymptotics for Total Cross Sections in N-body Problems. In "Spectral and Scattering Theory", 181-196, Lectures Notes in Pure and Applied Mathematic, vol. 161, Marcel Dekker, 1994.

[V] A. Vasy: Scattering matrices in many-body scattering, Commun. in Math. Phys. 200, 105-124, 1999.

[W] X.P.Wang: Total Cross Sections in N-body Problems: Finiteness and High Energy Asymptotics. Comm.Math.Phys. 156, 333-354, 1993.

[Y] D.R.Yafaev: The eikonal approximation and the asymptotics of the total scattering cross-section for the Schrödinger equation. Ann. Inst. Henri Poincar, vol. 40(4), 397-425, 1986. 